\title{
Exosomal miR-29b found in aqueous humour mediates calcium signaling in diabetic patients with cataract
}

\section{Chao Gao}

Eye and ENT Hospital of Fudan University

Xin Liu

Eye and ENT Hospital of Fudan University

Fan Fan

Eye and ENT Hospital of Fudan University

Jianing Yang

Eye and ENT Hospital of Fudan University

Xiyue Zhou

Eye and ENT Hospital of Fudan University

Hengjun Mei

Eye and ENT Hospital of Fudan University

\section{Xiaolei Lin}

Eye and ENT Hospital of Fudan University

Yi Luo ( $\nabla$ yeeluo1106@163.com )

Fudan University Eye Ear Nose and Throat Hospital Department of Ophthalmology

https://orcid.org/0000-0001-5971-1200

\section{Research article}

Keywords: Exosomes, miR-29b, diabetes and cataracts, Ca2+, CACNA1C

Posted Date: September 24th, 2020

DOl: https://doi.org/10.21203/rs.3.rs-34868/v2

License: (c) (1) This work is licensed under a Creative Commons Attribution 4.0 International License.

Read Full License 


\section{Abstract}

Background: $\mathrm{Ca}^{2+}$ was supposed to play an important role in the formation of cataract. Exosomal miR$29 \mathrm{~b}$ from aqueous humour may play an important role in the mechanism of diabetes and cataracts. The purpose of this study was to investigate the role of exosomal miR-29b and $\mathrm{Ca}^{2+}$ in regulating the function of human lens epithelial cells.

Methods: Exosomes were isolated from human aqueous humour by ultracentrifugation, and visualized by nanoparticle tracking and transmission electron microscopy. Exosomal miRNA sequencing was performed to identify differentially expressed miRNAs between diabetes and cataracts group (DMC) and age-related cataracts group (ARC). TargetScan was used to predict potential target of certain miRNA. The expression of CACNA1C mRNA was determined by quantitative real-time PCR and CACNA1C protein was determined by Western blotting. Concentration of $\mathrm{Ca}^{2+}$ of human aqueous humour and cell culture supernatant was detected by the Calcium Assay Kit. Cell Counting Kit-8 was used to determine cell viability.

Results: Exosomes were isolated from human aqueous humour, which had a typical cup-shaped phenotype and a particle size distribution in accordance with micro extracellular vesicles. Exosomal miRNA sequencing revealed that miR-29b was significantly downregulated in diabetes and cataracts group (DMC) compared with age-related cataracts group (ARC). $\mathrm{Ca}^{2+}$ concentration of human aqueous humour in DMC was higher than that in ARC. Cell culture supernatant transfected with miR-29b inhibitors had a higher concentration of $\mathrm{Ca}^{2+}$ than that transfected with miR-29b mimics. miR-29b reduced the viability of human lens epithelium cells (HLECs) by up-regulating CACNA1C expression.

Conclusions: Exosomes isolated from human aqueous humour contained abundant miRNAs. A significantly expressed miRNA, miR-29b, could affect the concentration of $\mathrm{Ca}^{2+}$ and regulate HLEC processes by up-regulating CACNA1C.

\section{Background}

Opacification of crystalline lenses is a disease that can be caused by many factors and is called cataract. Diabetes is a complex metabolic disorder that also involves small blood vessels, often causing widespread damage to tissues, including eyes. Cataracts are a common ocular complication of diabetes. Bilateral cataracts occasionally occur with rapid onset in severe juvenile diabetes, which is called true diabetic cataract. However, true diabetic cataracts are rare. Senile cataract in people with diabetes, which is called diabetes and cataracts in our study, is more common. The potential mechanisms for the pathogenesis of diabetes and cataracts are complicated and include the p38-MAPK signalling pathway ${ }^{[1,2]}$, polyol pathway ${ }^{[3]}$, and changes in inflammatory cytokines ${ }^{[4-10]}$. However, the precise mechanism of diabetes and cataracts remains unclear. 
$\mathrm{Ca}^{2+}$ exists in the endocytoplasmic reticulum and plays an important role in the processing of polypeptide chains in protein translation and post-translational processing. It was reported that abnormal distribution of $\mathrm{Ca}^{2+}$ could lead to dysfunction of the endocytoplasmic reticulum and mitochondria, thus causing some metabolic diseases such as diabetes ${ }^{[11]}$. Previous studies have reported that $\mathrm{Ca}^{2+}-\mathrm{CaM}$ abnormalities exist in cataracts, and it was found that L-type calcium channels were extensively distributed in lens epithelial cells, and the inhibition of L-type calcium channels could lead to the formation of cortical cataract ${ }^{[12]}$. L-type calcium channels were partially translated from the gene CACNA1C, which was also found to be mutated in diabetes and cataracts ${ }^{[13]}$. Given the critical role of $\mathrm{Ca}^{2+}$ in lens epithelial cells, further investigation on the role of $\mathrm{Ca}^{2+}$ in diabetes and cataracts is required.

Exosomes are micro extracellular vesicles $(30-150 \mathrm{~nm})$ that can be secreted by almost all types of cells and contain nucleus acids, proteins and lipids ${ }^{[14]}$. Exosomes have been widely studied in diabetes, neural diseases, tumours, cardiovascular diseases, etc ${ }^{[15-25]}$. A previous study reported that there were abundant exosomes in the human aqueous humour ${ }^{[26]}$. Exosomal microRNAs (miRNAs) play important roles in the mechanisms of diabetes ${ }^{[27-29]}$ and diabetic retinopathy ${ }^{[30-32]}$. However, the function and characteristics of exosomes and miRNAs in diabetes and cataracts remains unknown.

In this study, we found that decreased expression of miR-29b could upregulate the expression of CACNA1C, increase the concentration of $\mathrm{Ca}^{2+}$, and affect the apoptosis of human lens epithelial cells (HLECs) in patients with diabetes and cataracts. The role of miR-29b and calcium signalling in diabetic cataracts was not reported before, which might suggest a potential role of exosomal miRNAs in the pathogenesis of diabetes and cataracts.

The purpose of the study was to reveal the potential function of exosomal miRNAs found in aqueous humour on calcium signaling in diabetic patients with cataract.

\section{Methods}

\section{Ethics statement}

The use of human aqueous humour (AH) samples from cataract eyes during surgery was approved by the Institutional Review Board of Eye and ENT hospital of Fudan University. This study was performed in accordance with the tenets of the Declaration of Helsinki for research involving human subjects. Written informed consent was obtained from every enrolled participant.

\section{Collection of $\mathrm{AH}$ and human lens epithelium}

We collected $\mathrm{AH}$ and human lens epithelium from 36 patients with diabetes and cataracts (age from 4576 years old, fasting glucose from $5.2-8.2 \mathrm{mmol} / \mathrm{L}$, free of other ocular diseases, and lenticular opacity ranging from C3-4, NO2-3, NC2-3, and P1-3 by LOCSIII) and 43 patients with age-related cataracts (age from 62-85 years old, fasting glucose from 5.0-6.2 mmol/L, free of other ocular diseases, C3-4, NO2-3, 
NC2-3, and P1-2) before cataract surgery at Eye and ENT Hospital of Fudan University. AH samples were obtained before the collection of lens epithelium samples. The lens epithelium samples were acquired by intact continuous curvilinear capsulorhexis during cataract surgery for ARC patients by the same experienced surgeon (Yi Luo). All AH and human lens epithelium samples were stored in a freezer at $-80^{\circ} \mathrm{C}$ until the next step.

3 Isolation of exosomes

The AH samples from 36 patients with diabetes and cataracts were pooled together as the DMC group, and the AH samples from 43 patients with age-related cataracts were pooled together as the ARC group. Exosomes were isolated using ultracentrifugation. Procedures were as followed: a) Take AH samples out and thaw in $27^{\circ} \mathrm{C}$ water bath. b) $4^{\circ} \mathrm{C}, 2000 \mathrm{~g}, 10 \mathrm{~min}$, and remove supernatant. c) $4^{\circ} \mathrm{C}, 10000 \mathrm{~g}, 30 \mathrm{~min}$, and take supernatant. d) $4^{\circ} \mathrm{C}, 110000 \mathrm{~g}, 75 \mathrm{~min}$, and discard supernatant. e) Resuspend pellet and filter with 0.22 um membrane. f) $4^{\circ} \mathrm{C}, 110000 \mathrm{~g}, 75 \mathrm{~min}$, and abandon supernatant.

4 Transmission electron microscopy (TEM) and nanoparticle tracking analysis (NTA) of AH exosomes

Purified exosomes were diluted in PBS. $5 \mu \mathrm{L}$ samples were absorbed onto copper grids and dried for 5 minutes at room temperature. After that, a drop of $2 \%$ uranyl acetate solution was added for 20 minutes, and the sample was air-dried and examined by TEM (Tecnai G2 Spirit BioTwin, FEI, USA). Particle size, concentration, and distribution of exosomes were determined by NTA (ZetaView, Particle Matrix, Germany).

5 Exosomal RNA extraction and miRNA sequencing analysis

Exosomal RNA was extracted from the DMC group and the ARC group using the miRNeasy Micro Kit (217084, QIAGEN, Germany) according to the manufacturer's guidelines. RNA libraries were prepared and sequenced on an Illumina HiSeq 2500 platform. Read counts were obtained by FeatureCounts software. FastQC software was used for quality control. Additionally, we used Cutadapt software to remove lowquality reads and high-quality reads were used to analyse miRNAs by mapping to the human reference genome using Bowtie software. A fold change $>1.2$ or $<0.83$ was considered to indicate differentially expressed miRNAs by DESeq2.

6 RNA extraction of epithelium samples and quantitative real-time PCR (qRT-PCR)

Epithelium samples from 43 patients with age-related cataracts were classified as ARC group. Epithelium samples from 36 patients with diabetes and cataracts were classified as DMC group. In each group, 4 to 5 epithelium samples were pooled together to obtain enough RNA. Total RNA from all epithelium samples was extracted using TRIzol reagent (Invitrogen, Carlsbad, CA, USA) and reverse transcribed with the RT reagent Kit (Takara Bio, Inc, Japan) according to the manufacturer's protocol. Expression of mRNAs was detected using the SYBR Green detection kit (Takara, Japan) on the LightCycler 480II Real-Time PCR 
System (Roche, Switzerland). GAPDH was detected as the internal control. RNA expression was determined by the $2^{-\triangle \Delta C T}$ method.

7 Human lens epithelial cell culture and transfection

HLECs were cultured in 35-mm culture Petri dishes with growth medium containing DMEM (Gibco, USA) with $10 \%$ foetal bovine serum (FBS, Gibco, USA). MiR-29b mimics ( $50 \mathrm{nM}$ ) and inhibitors (100 nM) were transfected into HLECs when the cells covered $70-80 \%$ of the entire dish.

8 Examination of $\mathrm{Ca}^{2+}$ of $\mathrm{AH}$ samples and cell culture supernatant

Concentration of $\mathrm{Ca}^{2+}$ of $\mathrm{AH}$ samples and cell culture supernatant was detected by using Calcium Assay Kit (Colorimetric) from Abcam (Cambridge, MA, USA) according to the manufacturer's protocol. We added $50 \mu \mathrm{L}$ of $\mathrm{AH}$ samples and cell culture supernatant to each well of the 96 -well plates. Then $90 \mu \mathrm{L}$ of the Chromogenic Reagent and $60 \mu \mathrm{L}$ of Calcium Assay Buffer were added into each well for 10 minutes at room temperature protected from light. Absorbance was measured at a wavelength of $575 \mathrm{~nm}$ using an automatic microplate reader (Tecan, Switzerland). Concentration of $\mathrm{Ca}^{2+}$ was equivalent to Abs/Vol (ug/ $\mu \mathrm{L}$ ). (Abs referred to absorbance of AH samples and cell culture supernatant, Vol referred to the volume of $\mathrm{AH}$ samples and cell culture supernatant added to each well)

9 Cell Counting Kit-8 (CCK-8) cell proliferation and cytotoxicity assay

Cell viability was determined by using CCK-8 kit (Dojindo, Japan) according to the manufacturer's protocol. Transfected cells were plated onto 96-well plates and cultured for 24 hours. CCK-8 $(10 \mu \mathrm{L})$ was then added for 1 hour at $37^{\circ} \mathrm{C}$. We used Tert-butyl hydroperoxide solution (TBHP) as an oxidative stimulus. Absorbance was measured at a wavelength of $450 \mathrm{~nm}$ using an automatic microplate reader (Tecan, Switzerland). The cell viability was equivalent to (At-Ab)/(Ac-Ab). (At referred to absorbance of transfected cell groups, Ac referred to absorbance of controlled groups, Ab referred to absorbance of blank groups)

10 Western blot

Protein was extracted by RIPA lysis buffer (Biotech Well, Shanghai, China). Equal amounts of proteins were resolved by SDS-PAGE using $5 \%$ acrylamide-containing gels, followed by electrophoretic transfer to PVDF membranes. The membranes were blocked with transfer buffer (Biotech Well, Shanghai, China) and incubated overnight with the monoclonal primary antibodies at a 1:200 dilution, followed by secondary antibodies at a 1:2000 dilution. The signaling of western blotting was then observed using ECL prime reagents (Biotech Well, Shanghai, China) and scanned using a Peiqing automatic gel imaging analysis system (Shanghai, China). The L-VOCC polyclonal antibody (21774-1-AP) was purchased from Proteintech Group (USA). The anti-GAPDH antibody and the goat anti-rabbit IgG $(\mathrm{H}+\mathrm{L})$ secondary antibody were purchased from Biotech Well (Shanghai, China). 
Potential targets of miR-29b were predicted by the Targetscan database (http://www.targetscan.org) ${ }^{[33]}$.

13 Statistical analysis

All data are shown as the mean $\pm S D$, and experiments were repeated three times. Statistical significance was determined by two-tailed Student's $t$-test, One-way ANOVA or chi-square test using IBM SPSS 21.0(USA). $P$-value $<0.05$ was considered statistically significant.

\section{Results}

1 Baseline characteristics in DMC and ARC groups

79 eyes were enrolled in the two groups, among which there were 36 eyes in DMC group and 43 eyes in ARC group. In DMC group, there were 22 males and 14 females, 17 right eyes and 19 left eyes. Mean age and mean fasting glucose of DMC group were $68.11 \pm 1.34$ year-old and $6.31 \pm 1.34 \mathrm{mmol} / \mathrm{L}$. In ARC group, there were 21 males and 22 females, 21 right eyes and 22 left eyes. Mean age and mean fasting glucose of ARC group were $69.07 \pm 0.82$ year-old and $5.55 \pm 0.06 \mathrm{mmol} / \mathrm{L}$. There were no statistical significance in the gender, right or left eye, and age between the two groups (Table 1). The fasting glucose between the two groups was statistically significant (Table 1 ).

\begin{tabular}{|c|c|c|c|}
\hline & DMC group & ARC group & $P$ value \\
\hline Number & 36 & 43 & - \\
\hline Age (year-old) & $68.11 \pm 1.34$ & $69.07 \pm 0.82$ & 0.530 \\
\hline \multicolumn{4}{|l|}{ Gender } \\
\hline Male & 22 & 21 & \multirow[t]{2}{*}{0.365} \\
\hline Female & 14 & 22 & \\
\hline \multicolumn{4}{|l|}{ Right or left eye } \\
\hline Right eye & 17 & 21 & \multirow[t]{2}{*}{0.886} \\
\hline Left eye & 19 & 22 & \\
\hline Fasting glucose(mmol/L) & $6.31 \pm 1.34$ & $5.55 \pm 0.06$ & $<0.001$ \\
\hline
\end{tabular}

Table 1. Baseline characteristics in DMC and ARC groups, including numbers of patients, age, gender, right or left eye, and fasting glucose

2 Cup-shaped phenotype observed using TEM and particle size distribution of exosomes using NTA

To identify the characteristics of exosomes, we used TEM to observe the morphology of exosomes isolated from aqueous humour, and we observed a typical cup-shaped phenotype in both groups (Figure 1a). 
The particle diameter was $129.8 \mathrm{~nm}$ in the ARC group and $116.3 \mathrm{~nm}$ in the DMC group. Particle sizes were mainly distributed at $80-120 \mathrm{~nm}$, and the concentration reached more than $\mathrm{E}+10$ particles $/ \mathrm{ml}$ in both groups (Figure 1b).

3 Differential expression of miRNAs in AH samples of the DMC and ARC groups

miRNA sequencing was used to examine the expression of miRNAs in AH samples of the DMC and ARC groups. A total of 552 miRNAs were obtained from all samples. Of them, 119 miRNAs were unchanged; 295 miRNAs were upregulated and 138 miRNAs were downregulated in AH samples of the DMC group compared with the ARC group (fold change $>1.2$ in upregulated miRNAs, and fold change $<0.833$ in downregulated miRNAs) (Table S1). Among the 138 downregulated miRNAs, miR-29b was highly expressed in the DMC group compared with the ARC group (with fold change=0.286) (Figure 2).In addition, CACNA1C was a potential target of miR-29b through the TargetScan website. We thus investigated the role of miR-29b in the regulation of HLECs function. Part of differentially expressed miRNAs were shown in Figure 3.

4 Differential CACNA1C expression between DMC and ARC epithelium samples

CACNA1C mRNA expression was upregulated in DMC epithelium samples compared with that in ARC epithelium samples by qRT-PCR, and the difference was statistically significant (Figure 3 ).

5 Different concentration of $\mathrm{Ca}^{2+}$ of $\mathrm{AH}$ samples in the $\mathrm{DMC}$ and $\mathrm{ARC}$ groups

To determine the effect affected by the different expression of CACNA1C, we detected the concentration of $\mathrm{Ca}^{2+}$ of $\mathrm{AH}$ samples in two groups. Mean concentration of $\mathrm{Ca}^{2+}$ of $\mathrm{AH}$ samples was $0.075 \pm 0.005$ $\mathrm{ug} / \mathrm{uL}$ in the DMC group and $0.06 \pm 0.004 \mathrm{ug} / \mathrm{uL}$ in the ARC group. The concentration of $\mathrm{Ca}^{2+}$ of $\mathrm{AH}$ samples was higher in the DMC group than that in the ARC group ( $25 \%$ more), and the difference was statistically significant (Figure 4).

6 MiR-29b mimics and inhibitors significantly changed CACNA1C expression in HELCs

To determine whether exosomal miR-29b influenced the expression of CACNA1C in HELCs, we used miR$29 \mathrm{~b}$ mimics and inhibitors to transfect HLECs and then detect the expression of CACNA1C mRNA of HLECs. miR-29b mimics significantly downregulated CACNA1C expression in HELCs, while miR-29b inhibitors significantly upregulated CACNA1C expression in HELCs (Figure 5a). Additionally, western blot analysis showed that transfection of miR-29b mimics led to a reduction in CACNA1C expression. By contrast, transfection of miR-29b inhibitors led to increased CACNA1C expression (Figure 5b). The results were consistent with the results observed in epithelium samples.

7 Different concentration of $\mathrm{Ca}^{2+}$ of cell culture supernatant transfected by miR-29b mimics and inhibitors 
To determine whether different expression of CACNA1C regulated by miR-29b affected the concentration of $\mathrm{Ca}^{2+}$, we then detect the concentration of $\mathrm{Ca}^{2+}$ of cell culture supernatant transfected by miR-29b mimics and inhibitors. Mean concentration of $\mathrm{Ca}^{2+}$ of cell culture supernatant was $0.09 \pm 0.005 \mathrm{ug} / \mu \mathrm{L}$ in the control group, and $0.078 \pm 0.01 \mathrm{ug} / \mu \mathrm{L}$ transfected by miR-29b mimics, and $0.106 \pm 0.002 \mathrm{ug} / \mu \mathrm{L}$ transfected by miR-29b inhibitors. The concentration of $\mathrm{Ca}^{2+}$ was higher in the cell culture supernatant transfected by miR-29b inhibitors than that in normal cell culture supernatant. The concentration of $\mathrm{Ca}^{2+}$ was $26.4 \%$ higher in the cell culture supernatant transfected by miR-29b inhibitors than that in the cell culture supernatant transfected by miR-29b mimics (Figure 6). The result was similar to that of $\mathrm{AH}$ samples.

8 The role of miR-29b in the regulation of cell viability of HLECs

We further investigated the effect of miR-29b on the viability of HLECs. Both under normal conditions and under oxidative stress, miR-29b inhibitors significantly reduced the viability of HLECs compared with miR$29 \mathrm{~b}$ mimics, and the difference was statistically significant (Figure 7a, b). Under oxidative stress, the viability of HLECs transfected with miR-29b mimics was slightly reduced, but the difference was not statistically significant (Figure 7c). However, the viability of HLECs transfected with miR-29b inhibitors was significantly reduced, and the difference was statistically significant (Figure 7d).

\section{Discussion}

Cataracts are more common in patients with diabetes, the symptoms of which are similar to those of agerelated cataracts, while the progression is more rapid. Recently, exosomes have been widely studied in many fields, especially in diabetes. To reveal a possible mechanism of diabetes and cataracts, we investigate the role of exosomal miR-29b and $\mathrm{Ca}^{2+}$ in human lens epithelial cells.

$\mathrm{Ca}^{2+}$ is an important factor in life activity. More importantly, calcium might induce the conformational damages of A-crytallin and accelerate the development of the cataract ${ }^{[34]}$. However, the precise mechanism of how $\mathrm{Ca}^{2+}$ plays its role still remained unclear. In our study, we detected the concentration of $\mathrm{Ca}^{2+}$ of $\mathrm{AH}$ samples of $\mathrm{DMC}$ and $\mathrm{ARC}$, and found that the concentration of $\mathrm{Ca}^{2+}$ of $\mathrm{AH}$ samples is higher in the DMC than that in the ARC, showing a potential relationship among $\mathrm{Ca}^{2+}$ and diabetes and cataracts. Besides, CACNA1C mRNA expression is up-regulated in DMC epithelium samples compared with that in ARC epithelium samples, which partially translates L-type calcium channels that might lead to the different concentrations of $\mathrm{Ca}^{2+}$ in two groups.

Exosomes have been widely studied in diabetes, neural diseases, tumors, cardiovascular diseases, etc ${ }^{[15-}$ 25]. Abundant exosomal miRNAs were found in human aqueous humour, containing miR-486, miR-204, miR-184, etc ${ }^{[26]}$. Exosomal miRNAs played important roles in the mechanisms of diabetes ${ }^{[27-29]}$. In diabetes-associated ocular diseases (such as diabetic retinopathy), exosomal miRNAs play an important role by affecting the integrity of vascular endothelia ${ }^{[31]}$. It was also reported that pancreatic $\beta$ cell derived 
exosomal miR-15a might damage the retina by targeting Akt3 and causing diabetic retinopathy ${ }^{[30]}$. In addition, exosomes of plasma carrying IgG can damage retinal vessels ${ }^{[32]}$, and retinal pigment epithelium (RPE) cell-derived exosomes can participate in immunoregulation by killing targeted monocytes ${ }^{[35,36]}$, thus causing diabetic retinopathy. In addition, miRNAs play important roles in regulating the function of coding genes. Down-regulation of miR-2113 inhibits high glucose-induced mesenchymal activation and fibrosis ${ }^{[37]}$. MiR-30a combined with circHIPK3 could regulate the expression of VEGFC, FZD4 and WNT2, thus changing the viability and apoptosis of retinal pigmented epithelial cells ${ }^{[38]}$. More importantly, our previous study showed up-regulation of miR-193a caused by down-regulation of circHIPK3 could regulate the formation of cataract by targeting CRAYY ${ }^{[39]}$. However, there are few studies reporting the function and characterization of exosomes and miRNAs in diabetes and cataracts.

In our previous study, we used bioinformatics to analyse the correlating functions of the differential expressed miRNAs, and found that the coding genes were mainly associated with proteoglycans in cancer adjacent to the up-regulated miRNAs and AGE-RAGE signalling pathway in diabetic complications adjacent to the down-regulated miRNAs. Among the up-regulated exosomal miRNAs, miR-551b was highly expressed and could down-regulate CRYAA expression, thus affecting the viability and apoptosis of HLECs ${ }^{[40]}$.

In this study, we assessed aqueous humour as a microenvironment of lens epithelial cells and focused on the influence of aqueous humour on the crystalline lens and sequenced exosomal miRNAs between DMC and ARC groups. Therefore, we isolated exosomes of aqueous humour by ultracentrifugation and identified the exosomes through NTA and TEM. We then sequenced exosomal miRNA and revealed that miR-29b is differentially downregulated in patients with diabetes and cataracts and compared the data with that from age-related cataracts. MiR-29b was previously reported to be significantly changed across the diabetes spectrum and associated with measures of pancreatic islet $\beta$ cell function and glycemic control ${ }^{[41]}$. In culturing trancfected cells, the concentration of $\mathrm{Ca}^{2+}$ was $26.4 \%$ more in the cell culture supernatant transfected by miR-29b inhibitors than that in the cell culture supernatant transfected by miR-29b mimics, which almost equivalent to that of AH samples (25\%). The results in our study revealed that the downregulation of miR-29b could cause the upregulation of CACNA1C expression and an increase of concentration of $\mathrm{Ca}^{2+}$, which results in a lower cell viability of human lens epithelium cells. Besides, HELCs transfected with miR-29b inhibitors are more sensitive to oxidative stress. Here, we provide a novel mechanism that the function of HLECs in diabetes and cataracts might be regulated through exosomal miR-29b/CACNA1C/Ca ${ }^{2+}$ (Figure 8).

\section{Conclusions}

Exosomes isolated from human aqueous humour contained abundant miRNAs. A significantly expressed miRNA, miR-29b, could affect the concentration of $\mathrm{Ca}^{2+}$ and regulate HLEC processes by up-regulating CACNA1C. 


\section{Declarations}

\section{Ethics approval and consent to participate}

The use of human aqueous humour (AH) samples from cataract eyes during surgery was approved by the Institutional Review Board of Eye and ENT hospital of Fudan University. This study was performed in accordance with the tenets of the Declaration of Helsinki for research involving human subjects. Written informed consent was obtained from every enrolled participant.

\section{Consent for publication}

Not applicable.

\section{Availability of data and material}

All data generated or analysed during this study are included in this published article and its supplementary files.

\section{Competing interests}

There is no competing interests in this study.

\section{Funding}

This work was supported by the National Natural Science Foundation of China (81870645), including the fund of materials and the use of instruments mentioned in the study.

\section{Authors' contributions}

CG designed the study, conducted part of experiments, analyzed part of data, and was a major contributor in writing the article.

$\mathrm{XL}$ designed the study, conducted part of experiments, collected samples, analyze part of data, and write part of the article.

FF conducted part of experiments, collected samples, and write part of the article.

JY conducted part of experiments, helped collected samples, and proofread part of the article.

XZ conducted part of experiments, helped collected samples, and proofread part of the article.

HM conducted part of experiments, helped collected samples, and proofread part of the article.

XL conducted part of experiments, helped collected samples, and proofread part of the article. 
YL was the corresponding author, designed the study, proofread and approved the final version of the article.

\section{Acknowledgements}

Not applicable.

\section{Abbreviations}

\begin{tabular}{|ll|}
\hline DMC & Diabetes and cataracts group \\
\hline ARC & Age-related cataracts group \\
\hline HLECs & Human lens epithelial cells \\
\hline AH & Aqueous humour \\
\hline TEM & Transmission electron microscopy \\
\hline NTA & Nanoparticle tracking analysis \\
\hline CCK-8 & Cell Counting Kit-8 \\
\hline qRT-PCR & quantitative real-time PCR \\
\hline
\end{tabular}

\section{References}

1.Zhu G, Gong W, Li J, et al. LncRNA MALAT1 promotes the apoptosis and oxidative stress of human lens epithelial cells via p38MAPK pathway in diabetic cataract. Diabetes Res Clin Pract. 2018, 144: 314-321.

2.Qi M, Elion E A. MAP kinase pathways[J]. Journal of Cell Science, 2005, 118(16): 3569-3572.

3.Collins JG, Corder CN. Aldose reductase and sorbitol dehydrogenase distribution in substructures of normal and diabetic rat lens. Invest Ophthalmol Vis Sci. 1977 Mar;16(3):242-3.

4.Hailiang W, De-Kuang H, Xudong S, et al. Association between Aqueous Cytokines and Diabetic Retinopathy Stage. Journal of Ophthalmology. Volume 2017.

5.Takeuchi M, Sato T, Sakurai Y, Taguchi M, Harimoto K, Karasawa Y, et al. (2017) Association between aqueous humor and vitreous fluid levels of Th17 cell-related cytokines in patients with proliferative diabetic retinopathy. PLoS ONE. 12(5): e0178230.

6.Kuzmin A, Lipatov D, Chistyakov T, et al. Vascular Endothelial Growth Factor in Anterior Chamber Liquid Patients with Diabetic Retinopathy, Cataract and Neovascular Glaucoma. Ophthalmol Ther (2013) 2:4151 . 
7.Hamld S, Gul A, Hamld Q. Relationship of cytokines and AGE products in diabetic and non-diabetic patients with cataract. International Journal of Health Sciences, Qassim University, Vol. 10, No. 4.

8.Stephan Neumann, Jens Linek, Gerhard Loesenbeck, et al. TGF- $\beta 1$ serum concentrations and receptor expressions in the lens capsular of dogs with diabetes mellitus. Open Veterinary Journal, (2017), Vol. 7(1): 12-15.

9.Delongh R, Gordon-Thomson C, Chamberlain CG, et al. TGFbeta receptor expression in lens: Implication for differentiation and cataractogenesis. Exp Eye Res.(2001)72,649-659.

10.Lovicu FJ, Schulz MW, Hales AM, et al. TGFbeta induces morphological and molecular changes similar to human anterior subcapsular cataract. Br J Ophthalmol. 2002;86(2):220-226.

11.Ana Paula Arruda and Gökhan S. Hotamisligil. Calcium homeostasis and organelle function in the pathogenesis of obesity and diabetes. Cell Metab. 2015 September 1; 22(3): 381-397. doi:10.1016/j.cmet.2015.06.010.

12.Maddala R, Nagendran T, de Ridder GG, Schey KL, Rao PV (2013) L-Type Calcium Channels Play a Critical Role in Maintaining Lens Transparency by Regulating Phosphorylation of Aquaporin-0 and Myosin Light Chain and Expression of Connexins. PLoS ONE 8(5): e64676. doi:10.1371/journal.pone.0064676.

13.Chang $C$, Zhang $K$, Veluchamy A, et al. A genome-wide association study provides new evidence that CACNA1C gene is associated with diabetic cataract. Invest Ophthalmol Vis Sci. 2016;57:2246-2250.

14.He C, Zheng S, Luo Y, et al. Exosome Theranostics: Biology and Translational Medicine[J]. Theranostics, 2018, 8(1):237.

15.Samuelson I, Vidal-Puig AJ. Fed-EXosome: extracellular vesicles and cell-cell communication in metabolic regulation. Essays Biochem. 2018 May 15;62(2):165-175. doi: 10.1042/EBC20170087.

16.Liberman M, Marti LC. Vascular Calcification Regulation by Exosomes in the Vascular Wall. Adv Exp Med Biol. 2017;998:151-160. doi: 10.1007/978-981-10-4397-0_10.

17.Saeedi Borujeni MJ, Esfandiary E, Taheripak G, et al. Molecular aspects of diabetes mellitus: Resistin, microRNA, and exosome. J Cell Biochem. 2018 Feb;119(2):1257-1272. doi: 10.1002/jcb.26271.

18.Guay C, Regazzi R. Exosomes as new players in metabolic organ cross-talk. Diabetes Obes Metab. 2017 Sep;19 Suppl 1:137-146. doi: 10.1111/dom.13027.

19.Li J, Fu LZ, Liu L, et al. Glucagon-Like Peptide-1 (GLP-1) Receptor Agonist Liraglutide Alters Bone Marrow Exosome-Mediated miRNA Signal Pathways in Ovariectomized Rats with Type 2 Diabetes. Med Sci Monit, 2017; 23: 5410-5419. doi: 10.12659/MSM.906603. 
20.Poornima Venkat, Michael Chopp, Jieli Chen. Cell-Based and Exosome Therapy in Diabetic Stroke. STEM CELLS TRANSLATIONAL MEDICINE 2018;7:451-455.

21.Wang QH, Ding XQ, Zhen F, et al. Remedial applications of exosomes in cancer, infections and diabetes. Acta Poloniae Pharmaceutica ñ Drug Research, Vol. 74 No. 2 pp. 313ñ320, 2017.

22.Zhang C, Zhang K, Huang FF, et al. Exosomes, the message transporters in vascular calcification. J Cell Mol Med. 2018;1-10. doi: 10.1111/jcmm.13692.

23.K. Agarwal, M. Saji, S. M. Lazaroff, et al. Analysis of Exosome Release as a Cellular Response to MAPK Pathway Inhibition. Langmuir. 2015 May 19; 31(19): 5440-5448. doi:10.1021/acs. langmuir.5b00095.

24.Sean M. Davidson, Jaime A. Riquelme, Kaloyan Takov, et al. Cardioprotection mediated by exosomes is impaired in the setting of type II diabetes but can be rescued by the use of non-diabetic exosomes in vitro. J. Cell. Mol. Med. Vol 22, No 1, 2018 pp. 141-151. doi: 10.1111/jcmm.13302.

25.Lee MJ, et al. Exosomal biomarker development in metabolic diseases. Ann Pediatr Endocrinol Metab 2016;21:119-125.

26.W. Michael Dismuke, Pratap Challa, Iris Navarro, et al. Human Aqueous Humor Exosomes. Exp Eye Res. 2015 March ; 132: 73-77. doi:10.1016/j.exer.2015.01.019.

27.Marta Garcia-Contreras, Sanket H. Shah, Alejandro Tamayo, et al. Plasma-derived exosome characterization reveals a distinct microRNA signature in long duration Type 1 diabetes. Scientific Reports 7: 5998. doi:10.1038/s41598-017-05787-y.

28.Wang X, Huang W, Liu G, et al. Cardiomyocytes mediate anti-angiogenesis in type 2 diabetic rats through the exosomal transfer of miR-320 into endothelial cells. J Mol Cell Cardiol. 2014 September ; 74: 139-150. doi:10.1016/j.yjmcc.2014.05.001.

29.Wang X, Gu H, Huang W, et al. Hsp20-Mediated Activation of Exosome Biogenesis in Cardiomyocytes Improves Cardiac Function and Angiogenesis in Diabetic Mice. Diabetes 2016;65:3111-3128. DOI: $10.2337 / \mathrm{db} 15-1563$.

30.Kamalden TA, Macgregor-Das AM, Kannan SM, Dunkerly-Eyring B, Khaliddin N, Xu Z, Fusco AP, Yazib SA, Chow RC, Duh EJ et al: Exosomal MicroRNA-15a Transfer from the Pancreas Augments Diabetic Complications by Inducing Oxidative Stress. Antioxid Redox Signal 2017, 27(13):913-930.

31.Klingeborn M, Dismuke WM, Bowes RC, Stamer WD: Roles of exosomes in the normal and diseased eye. PROG RETIN EYE RES 2017, 59:158-177.

32. Huang C, Fisher KP, Hammer SS. Plasma Exosomes Contribute to Microvascular Damage in Diabetic Retinopathy by Activating the Classical Complement Pathway. Diabetes. 2018 Aug;67(8):1639-1649. doi: 
33.B. Yan, J.T. Guo, C.D. Zhu, L.H. Zhao, J.L. Zhao, miR-203b: a novel regulator of MyoD expression in tilapia skeletal muscle, The Journal of experimental biology, 216 (2013) 447-451.

34.Sadaf Saba, Maryam Ghahramani and Reza Yousefi, A Comparative Study of the Impact of Calcium Ion on Structure, Aggregation and Chaperone Function of Human A-crystallin and its Cataract- Causing R12C Mutant, Protein \& Peptide Letters (2017) 24: 1048.

35.Serra AM, Waddell J, Manivannan A, Xu H, Cotter M, Forrester JV: CD11 b+ bone marrow-derived monocytes are the major leukocyte subset responsible for retinal capillary leukostasis in experimental diabetes in mouse and express high levels of CCR5 in the circulation. AM J PATHOL 2012, 181(2):719727.

36.Knickelbein JE, Liu B, Arakelyan A, Zicari S, Hannes S, Chen P, Li Z, Grivel JC, Chaigne-Delalande B, Sen $\mathrm{HN}$ et al: Modulation of Immune Responses by Extracellular Vesicles From Retinal Pigment Epithelium. Invest Ophthalmol Vis Sci 2016, 57(10):4101-4107.

37.Xue LP, Fu XL, Hu M, et al. Rg1 inhibits high glucose-induced mesenchymal activation and fibrosis via regulating miR-2113/RP11-982M15.8/Zeb1 pathway. Biochem Biophys Res Commun. 2018 Jul 2;501(4):827-832. doi: 10.1016/j.bbrc.2018.04.055.

38.Shan K, Liu C, Liu BH, et al. Circulation. Circular Noncoding RNA HIPK3 Mediates Retinal Vascular Dysfunction in Diabetes Mellitus. 2017 Oct 24;136(17):1629-1642.

39.Liu X, Liu C, Liu B, et al. Circular RNA HIPK3 regulates human lens epithelial cells proliferation and apoptosis by targeting the miR-193a/CRYAA axis. Biochem Biophys Res Commun. 2018 Jun 27. pii: S0006-291X(18)31468-2. doi: 10.1016/j.bbrc.2018.06.149.

40.Gao C, Fan F, Liu X, et al. (2020) Exosomal miRNA Analysis of Aqueous Humour of Diabetes and Cataract Patients, Current Eye Research, DOI: 10.1080/02713683.2020.1797107

41.Yury O. Nunez Lopez, Gabriella Garufi and Attila A. Seyhan. Altered levels of circulating cytokines and microRNAs in lean and obese individuals with prediabetes and type 2 diabetes. Mol. BioSyst., 2017,13, 106-121.

\section{Figures}



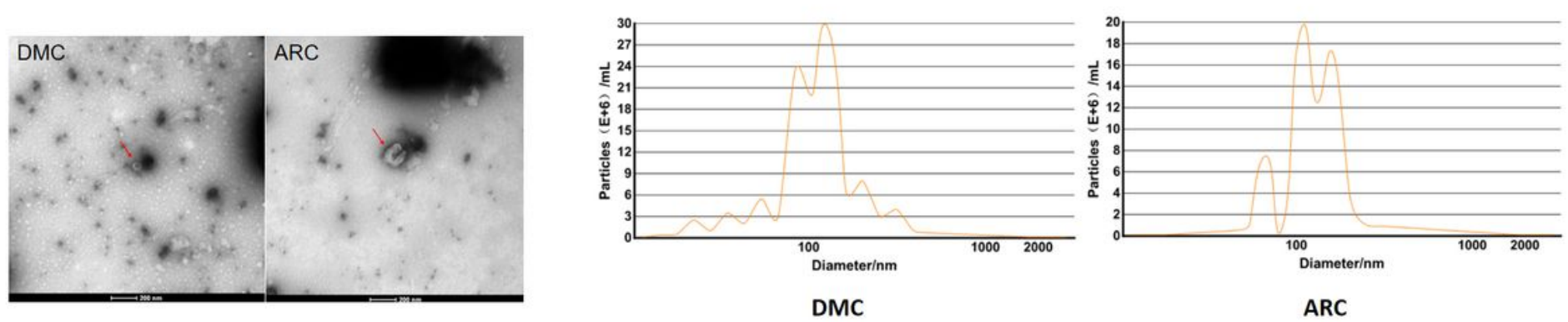

\section{Figure 1}

Identification of exosomes using TEM and NTA. a) Typical cup-shaped phenotype under TEM in the DMC and the ARC group. b) Particle size distribution in DMC and ARC group by NTA.

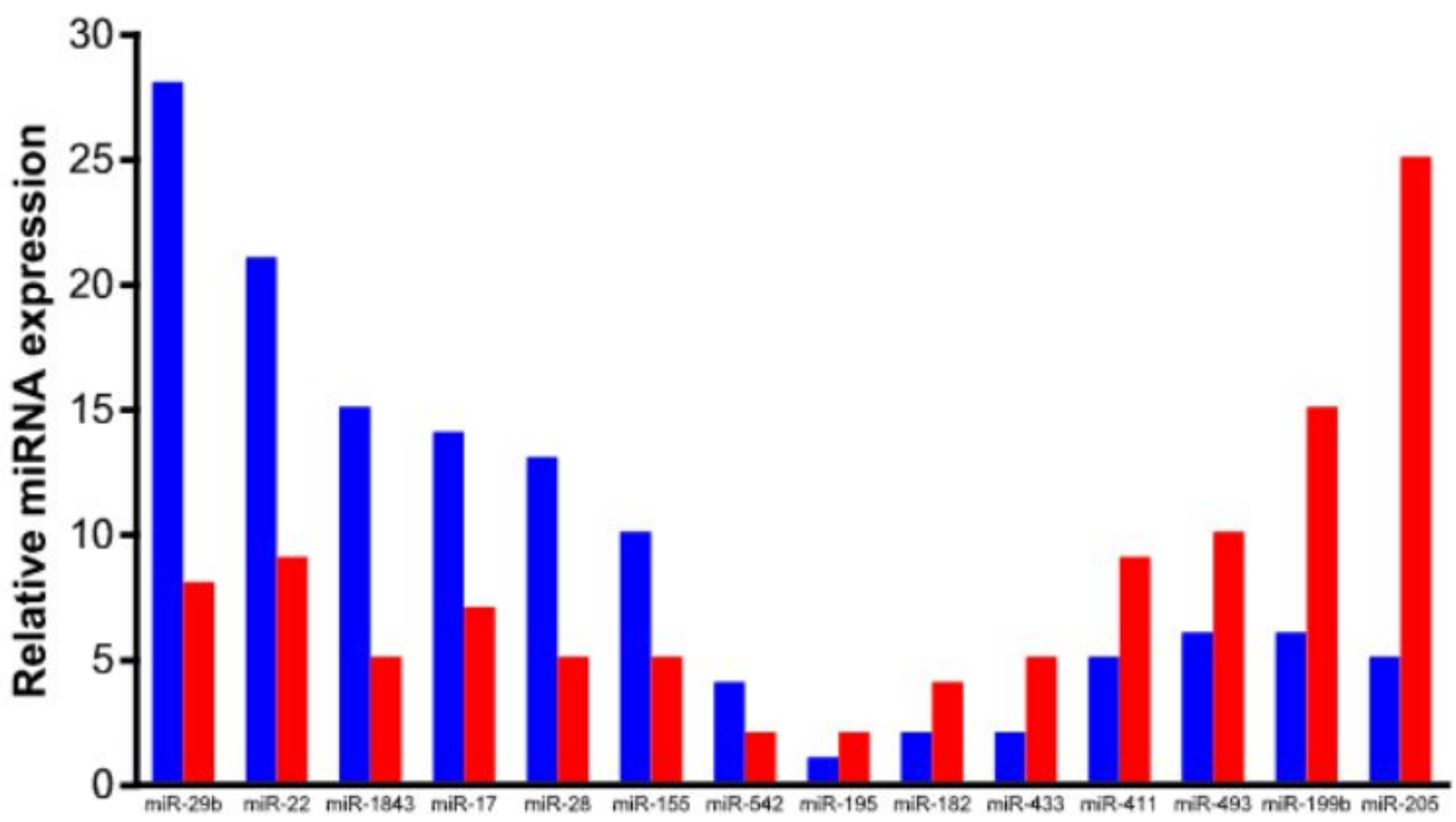

Figure 2

Part of downregulated and upregulated miRNA expression in the DMC group compared with the ARC group 


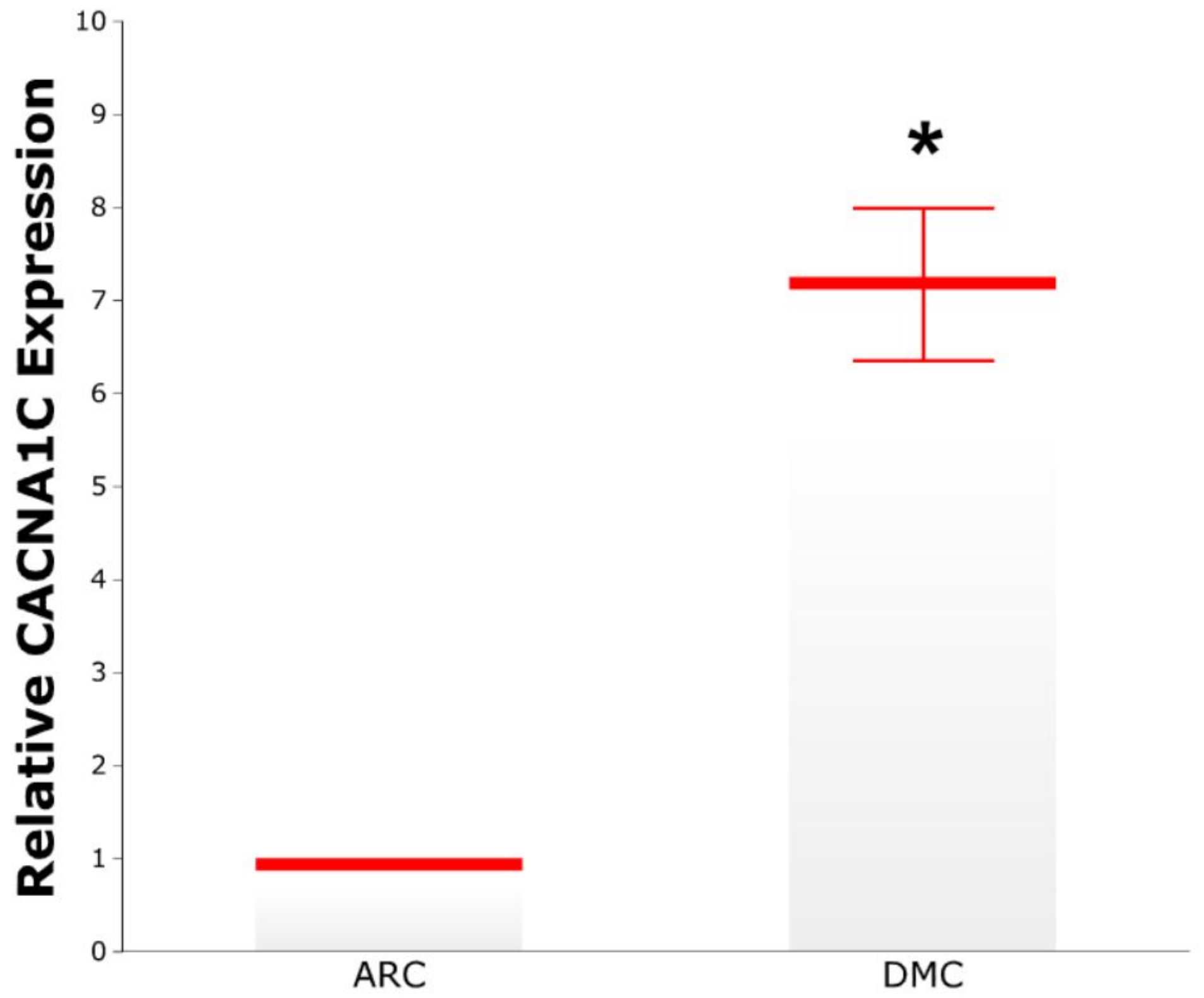

Figure 3

Expression of CACNA1C mRNA was detected by qRT-PCR. 


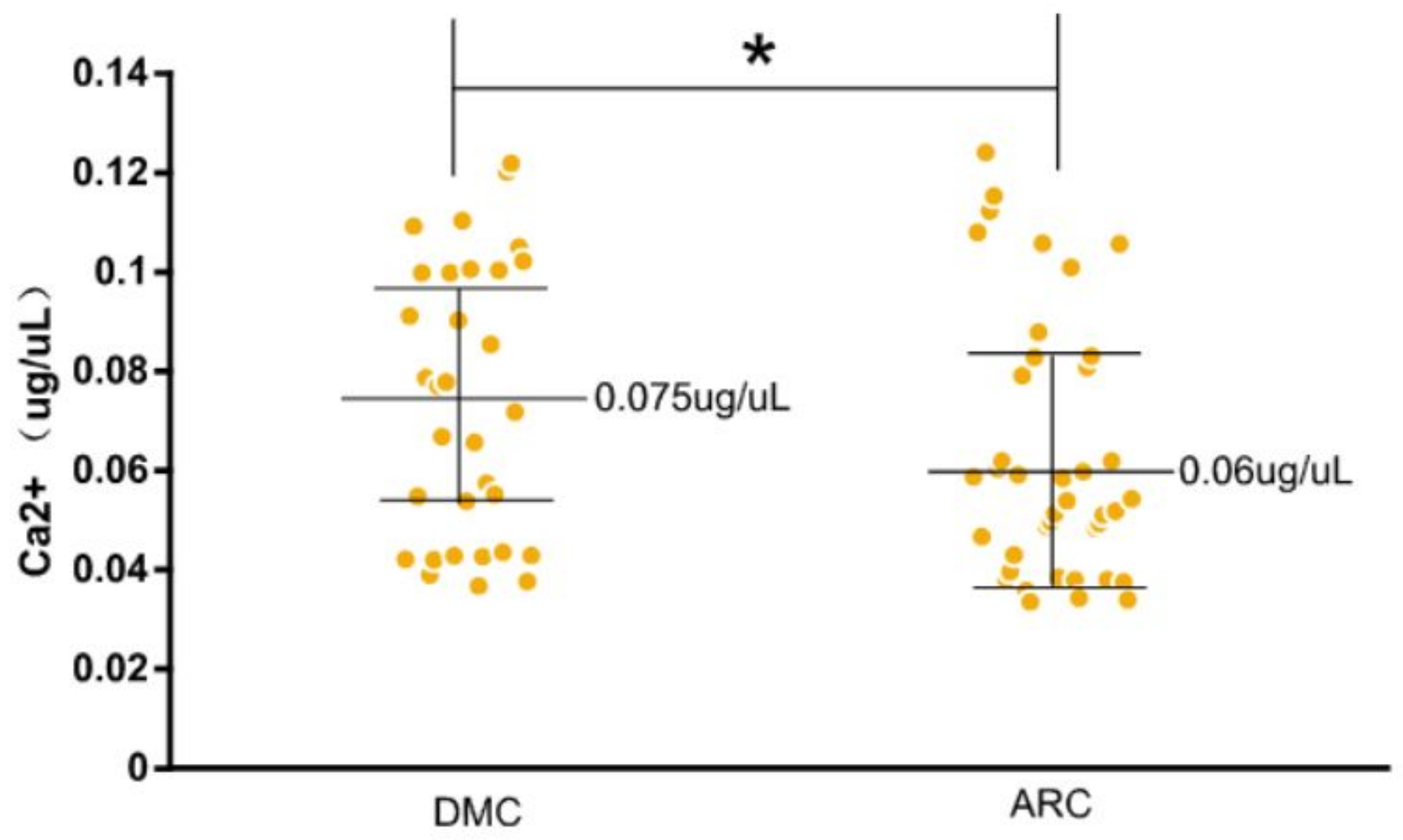

Figure 4

Concentration of $\mathrm{Ca} 2+$ of $\mathrm{AH}$ samples in the $\mathrm{DMC}$ and $\mathrm{ARC}$ groups
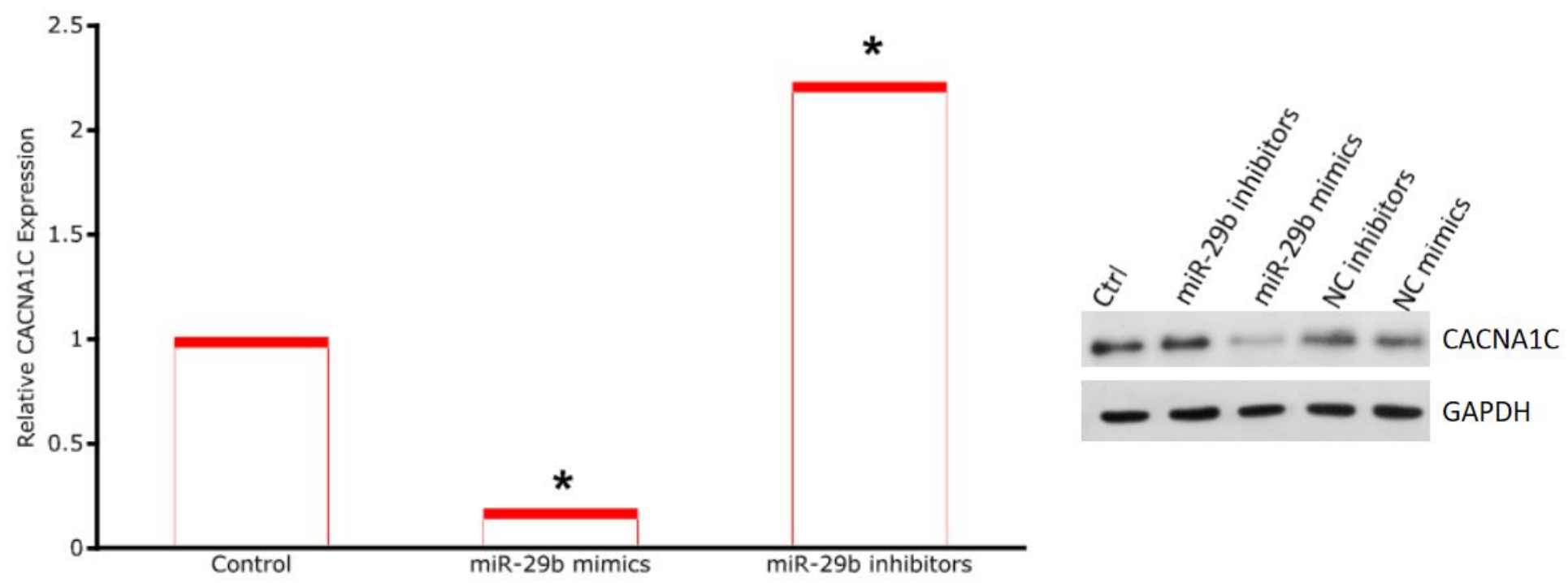

Figure 5

MiR-29b mimics and inhibitors changed CACNA1C expression in HELCs. (a) Expression of CACNA1C mRNA was affected by miR-29b mimics and inhibitors as demonstrated using qRT-PCR. (b) Protein levels of CACNA1C were affected by miR-29b mimics and inhibitors as shown using Western blotting. 


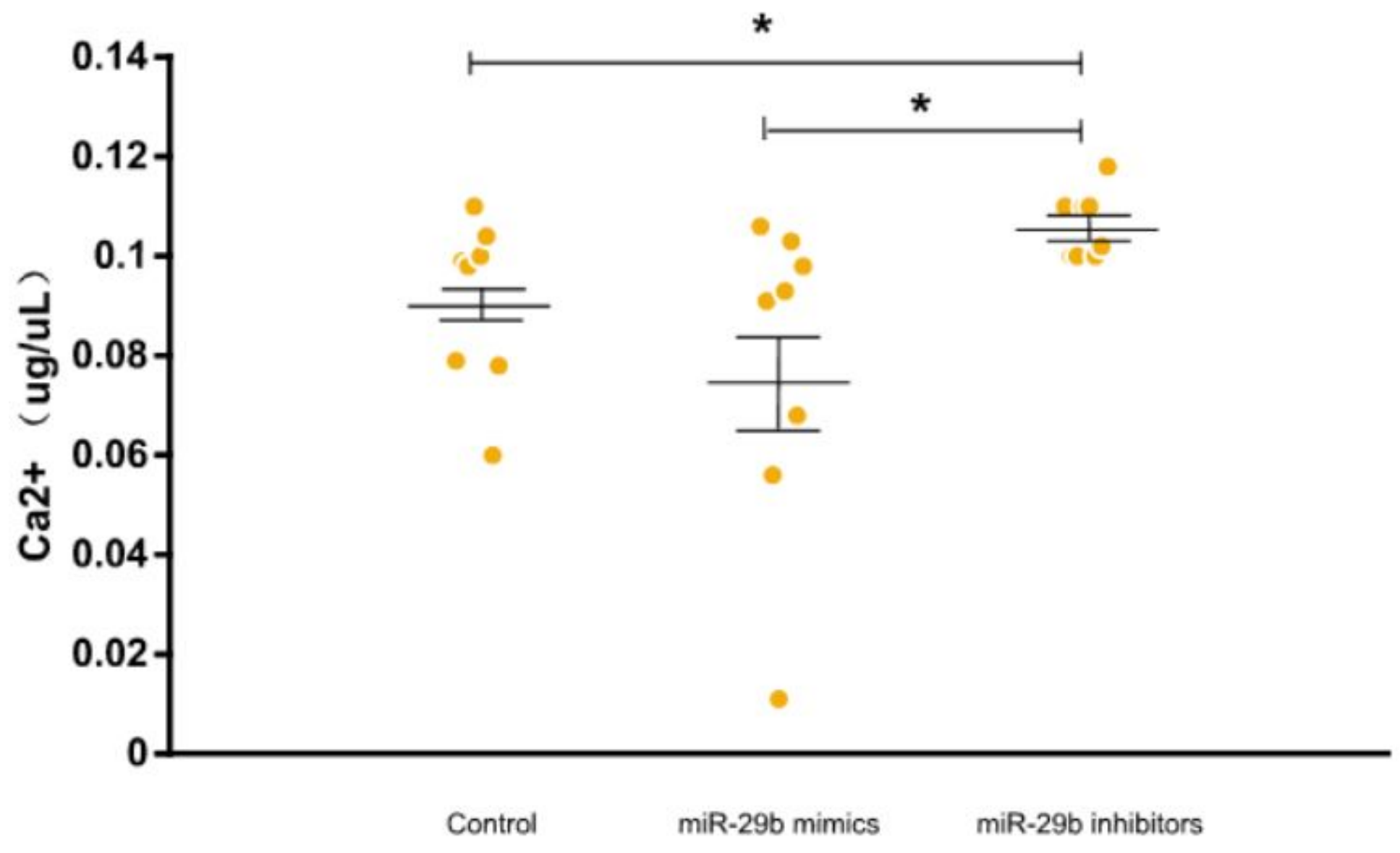

Figure 6

Concentration of $\mathrm{Ca} 2+$ in normal cell culture supernatant and in cell culture supernatant transfected by miR-29b mimics and inhibitors 

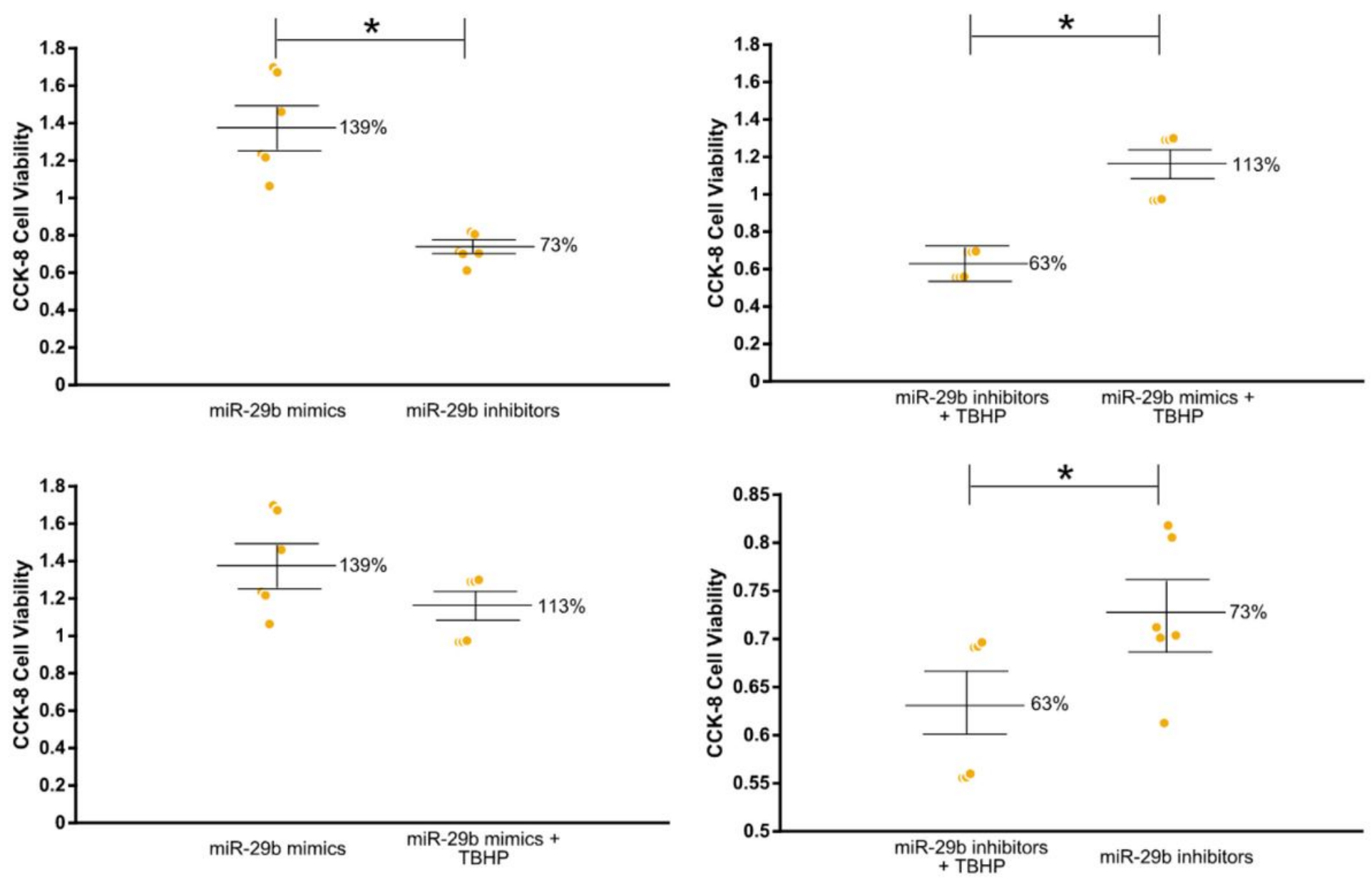

Figure 7

Cell viability was measured by CCK-8. (a) The viability of HLECs trancfected with miR-29b mimics and inhibitors in normal conditions. (b) The viability of HLECs trancfected with miR-29b mimics and inhibitors under oxidative stress. (c) The viability of HLECs trancfected with miR-29b mimics in normal conditions and under oxidative stress. (d) The viability of HLECs trancfected with miR-29b inhibitors in normal conditions and under oxidative stress. 


\section{Exosome}

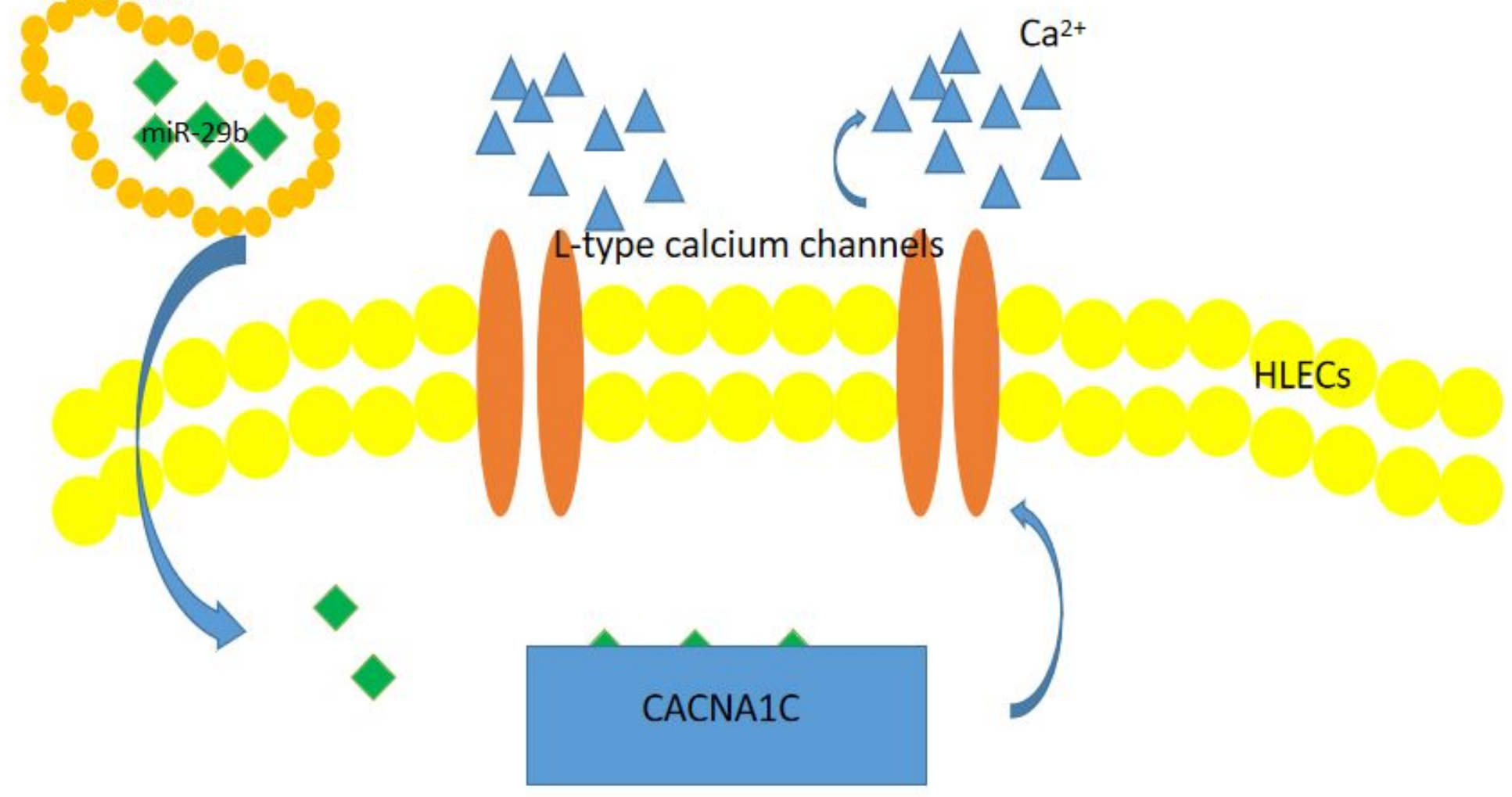

Figure 8

Possible regulation of function of HLECs through exosomal miR-29b/CACNA1C/Ca2+

\section{Supplementary Files}

This is a list of supplementary files associated with this preprint. Click to download.

- GAPDH.jpg

- LVOCC.jpg

- S1alldiffmiRNAs.xlsx

- S2upgenegoPathway.xlsx

- S3downgenegoPathway.xlsx

- S4upgeneKEGG.xIsx

- S5downgeneKEGG.xlsx 